\title{
Output decoupling property of planar flexure-based compliant mechanisms with symmetric configuration
}

\author{
Y. S. Du ${ }^{1,2}$, T. M. Li ${ }^{1,2}$, Y. Jiang ${ }^{1,2}$, and J. L. Zhang ${ }^{1,2}$ \\ ${ }^{1}$ Manufacturing Engineering Institute, Department of Mechanical Engineering, Tsinghua University, \\ Beijing, 100084, China \\ ${ }^{2}$ Beijing Key Lab of Precision/Ultra-precision Manufacturing Equipment and Control, Tsinghua University, \\ Beijing, 100084, China \\ Correspondence to: T. M. Li (litm@mail.tsinghua.edu.cn)
}

Received: 26 October 2015 - Revised: 15 January 2016 - Accepted: 1 February 2016 - Published: 10 February 2016

\begin{abstract}
This paper presents the output decoupling property of planar flexure-based compliant mechanisms with symmetric configuration. Compliance/stiffness modeling methods for flexure serial structures and flexure parallel structures are first derived according to the matrix method. Analytical model of mechanisms with symmetric configuration is then developed to analyze the output decoupling property. The proposed analytical model shows that mechanisms are output decoupled when they are symmetry about two perpendicular axes or when they are composed of either three or an even number of identical fundamental forms distributed evenly around the center. Finally, output compliances of RRR and 4-RRR compliant micro-motion stages are derived from the analytical model and finite element analysis (FEA). The comparisons indicate that the results obtained from the proposed analytical model are in good agreement with those derived from FEA, which validates the proposed analytical model.
\end{abstract}

\section{Introduction}

Flexure-based compliant mechanisms, which have advantages of no friction, no backlash, compact and monolithic structure, and ease of fabrication, are usually used as micropositioning stages (Acer and Sabanovic, 2011; Li et al., 2013; Yong and Lu, 2009). They can provide smooth motions through deflections of flexure hinges (Yong et al., 2008; Handley et al., 2004). Due to these advantages, coupled with nanometer positioning accuracy, flexure-based compliant mechanisms have been widely used in many industrial applications, such as scanning probe microscopy (Schitter et al., 2007; Leang and Fleming, 2009), lithography (Choi and Lee, 2005), nano-manipulation and manufacturing (Lai et al., 2012; Verma et al., 2005), and biological science (Ando et al., 2002; Kim et al., 2012). In addition, the flexure-based compliant positioning mechanisms with ultrahigh precision play more and more important roles in applications where a high resolution motion is desirable, such as the MEMS sensors and actuators, optical fiber alignment, and biological cell manipulation (Wang and Zhang, 2008; Li et al., 2012; Zubir et al., 2009).

Decoupling is the crucial property for parallel flexurebased compliant mechanisms. Generally, a decoupled parallel flexure-based compliant mechanism means that one actuator produces only one directional output motion without affecting the motion of other axes. The decoupled parallel flexure-based compliant mechanism should possess input decoupling and output decoupling properties (Li et al., 2012). The input decoupling can be defined as actuator isolation, which means that each actuator would not suffer extra loads induced by the actuation of other actuators. The output decoupling means that one actuator only drives the output platform in one axial direction. Generally, input coupling would impose undesired loads on the actuator and may even damage it. Output coupling would lead to complex kinematic models, thus making precise control difficult to be implemented.

Numerous studies have been carried out to design decoupled flexure-based compliant mechanisms. Among the investigations of flexure-based compliant parallel mecha- 
(a)

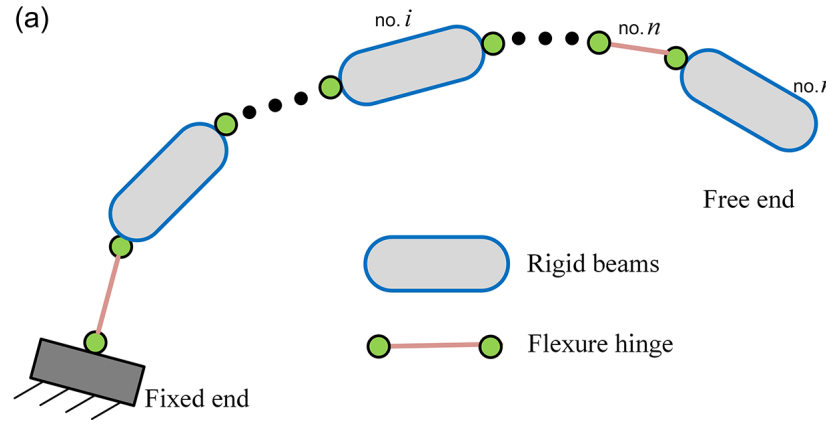

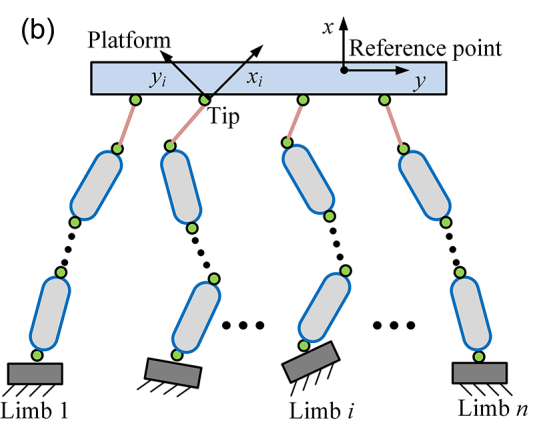

Figure 1. Flexure-based compliant mechanisms: (a) flexure serial structure; (b) flexure parallel structure.

nisms, the design of a totally decoupled one is first proposed in the literature (Awtar and Slocum, 2007). It presented parallel kinematic $X Y$ flexure mechanism designs based on systematic constraint patterns. These constraint arrangements allowed large primary motions and small error motions without running into over constraint problems. Kenton and Leang (2012) built a mechanism by serially stacking or nesting multiple one degree-of-freedom modules to eliminate the cross-axis coupling. Polit and Dong (2011) presented the design, analysis, fabrication, and testing of a highbandwidth parallel kinematic nano-positioning $X Y$ stage. The monolithic stage had two axes, and each axis was composed of a doubly clamped beam and a parallelogram hybrid flexure. The parallelogram hybrid flexures were used to decouple the actuation effect from the other axis. Thus, the mechanism design decoupled the motion in the $X$ and $Y$ directions and restricted parasitic rotations in the $X Y$ plane. $\mathrm{Li}$ and $\mathrm{Xu}(2008,2010)$ designed a decoupled $X Y$ flexure parallel kinematic manipulator. The output decoupling was allowed by the employment of compound parallelogram flexure. By contrast, the input decoupling was implemented by actuation isolation, which was enabled by the double compound parallelogram flexure with large transverse stiffness. Qin et al. (2014) introduced a 2-DOF monolithic mechanism. The statically indeterminate leaf parallelograms were used to provide the decoupling effect, and the displacement of piezoelectric actuator was amplified with a statically indeterminate lever mechanism. The cross-axis coupling ratio was experimentally measured to be below $1 \%$.

In the past decades, various decoupled compliant mechanisms have been developed. Nevertheless, there is rare analytical model which can prove the output decoupling property of flexure-based compliant mechanisms. Therefore, the present study addresses the output decoupling property of planar flexure-based compliant mechanisms composed of notch flexure hinges. Based on compliance/stiffness modeling methods, analytical model of mechanisms with symmetric configuration is derived. To verify the output decoupling property, the output compliances of the RRR (revolute-revolute-revolute) and 4-RRR compliant micro- motion stages are derived from the analytical model and FEA.

The remaining sections of this paper are organized as follows. In Sect. 2, compliance/stiffness modeling methods are derived, including the methods for flexure serial structures and flexure parallel structures. In Sect. 3, the output decoupling properties of the mechanisms, which are symmetric about two perpendicular axes or composed of several fundamental forms, are analyzed. And analytical model utilized for explaining the output decoupling property is derived. In Sect. 4, analytical model and FEA are used to analyze the output decoupling property of the RRR and 4-RRR micromotion stages. Finally, conclusions are drawn in Sect. 5.

\section{Compliance/stiffness modeling}

Flexure-based compliant mechanisms can be classified into two categories according to the kinematic structure: the flexure serial structure (Fig. 1a) and the flexure parallel structure (Fig. 1b). Serial structures are easier to design and have substantially decoupled degree-of-freedom, whereas the closedloop kinematic features of flexure parallel structures impart excellent performance in terms of high rigidity, high load carrying capacity, and high accuracy (Li et al., 2013; Pham and Chen, 2005). To analyze the output decoupling property of planar flexure-based compliant mechanism with symmetrical configuration, the compliance/stiffness modeling methods are derived firstly.

\subsection{Flexure serial structure}

A flexure serial structure comprised of several rigid beams and notch flexure hinges is illustrated in Fig. 1a. The relationship between the elastic deformations of a flexure member and the total deformation at the free end of the serial structure can be given as

$\boldsymbol{\delta}_{i}=\mathbf{J}_{d i} \boldsymbol{\delta}_{i}^{l}$,

where $\mathbf{J}_{d i}$ is the Jacobian matrix transforming a $3 \times 1$ vector $\delta_{i}^{l}$ representing the elastic deformations of flexure members 
to a $3 \times 1$ vector $\delta_{i}$ at the free end of the flexure serial structure.

According to the zero virtual work principle, the relationship between the external force at the free end and the reaction force at a flexure member can be given as

$\boldsymbol{F}_{i}^{l}=\mathbf{J}_{f i} \boldsymbol{F}$,

where $\mathbf{J}_{f i}$ is the Jacobian matrix transforming a $3 \times 1$ vector $\boldsymbol{F}$ of the external force at the free end to a vector of reaction force $\boldsymbol{F}_{i}^{l}$ at a flexure member.

The accumulation of both rotational and translational deformations at the free end of the flexure serial structure is

$\boldsymbol{\delta}=\sum_{i=1}^{n} \mathbf{J}_{d i} \boldsymbol{\delta}_{i}^{l}=\sum_{i=1}^{n} \mathbf{J}_{d i} \mathbf{C}_{i}^{l} \boldsymbol{F}_{i}^{l}=\left(\sum_{i=1}^{n} \mathbf{J}_{d i} \mathbf{C}_{i}^{l} \mathbf{J}_{f i}\right) \boldsymbol{F}$,

where $\mathbf{C}_{i}^{l}$ is the local compliance matrix established in the local frame attached to a flexure member.

Therefore, the compliance of the flexure serial structure is

$\mathbf{C}=\frac{\partial \boldsymbol{\delta}}{\partial F}=\sum_{i=1}^{n} \mathbf{J}_{d i} \mathbf{C}_{i}^{l} \mathbf{J}_{f i}=\mathbf{J}_{d} \mathbf{C}^{*} \mathbf{J}_{f}$

where

$\mathbf{J}_{d}=\left[\begin{array}{lllll}\mathbf{J}_{d_{1}} & \mathbf{J}_{d_{2}} & \mathbf{J}_{d_{3}} & \ldots & \mathbf{J}_{d_{n}}\end{array}\right]$

and

$\mathbf{C}^{*}=\operatorname{diag}\left[\mathbf{C}_{1} \mathbf{C}_{2} \mathbf{C}_{3} \ldots \mathbf{C}_{n}\right]$

For flexure serial structures, the compliance can be derived through the matrix method. The compliance shown in Eq. (4) can also be obtained through the screw theory based approach (Yu et al., 2011). It indicates that the proposed analysis of compliance modeling has close relations to the syntheses of compliant mechanism based on screw theory.

\subsection{Flexure parallel structure}

A flexure parallel structure can be considered as an infinitely rigid platform supported by $n$ limbs, as illustrated in Fig. 1b. We denote $\mathbf{K}_{i}$ as the stiffness matrix of limb $i$. The platform and the tips of the limbs have equivalent angular displacements. However, the linear displacements of the reference point of the platform are determined based on the linear and angular displacements of the tips of the limbs; hence,

$\delta=\mathbf{J}_{P i} \boldsymbol{\delta}_{i}$,

where $\delta$ and $\delta_{i}$ are, respectively, the $3 \times 1$ displacement vectors of the platform at the reference point and at the tip of the $i$ th limb, and $\mathbf{J}_{P i}$ is the amplification matrix of the displacement.
The $3 \times 1$ vector of the force and moment $F$ applied to the reference point of the platform is distributed to the tip of each limb as a $3 \times 1$ vector of force and moment $F_{i}$, hence,

$F=\sum_{i=1}^{n} \mathbf{J}_{f i} F_{i}=\sum_{i=1}^{n} \mathbf{J}_{f i} \mathbf{K}_{i} \boldsymbol{\delta}_{i}=\left(\sum_{i=1}^{n} \mathbf{J}_{f i} \mathbf{K}_{i} \mathbf{J}_{P i}^{-1}\right) \boldsymbol{\delta}$,

where $\mathbf{J}_{f i}$ is the transformation matrix when $F_{i}$ is moved from the tip of the $i$ th limb to the reference point.

Hence, the stiffness of the flexure parallel structure can be given as

$\mathbf{K}=\frac{\partial F}{\partial \boldsymbol{\delta}}=\sum_{i=1}^{n} \mathbf{J}_{f i} \mathbf{K}_{i} \mathbf{J}_{P i}^{-1}=\mathbf{J}_{F}^{*} \mathbf{K}^{*} \mathbf{J}_{P}^{*}$,

where

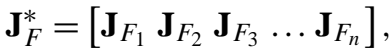

$\mathbf{J}_{p}^{*}=\left[\left(\mathbf{J}_{p_{1}}^{-1}\right)^{T}\left(\mathbf{J}_{p_{2}}^{-1}\right)^{T}\left(\mathbf{J}_{p_{3}}^{-1}\right)^{T} \ldots\left(\mathbf{J}_{p_{n}}^{-1}\right)^{T}\right]^{T}$,

and

$\mathbf{K}^{*}=\operatorname{diag}\left(\mathbf{K}_{1} \mathbf{K}_{2} \mathbf{K}_{3} \ldots \mathbf{K}_{n}\right)$.

Obviously, it is more convenient to use the compliance matrices for the flexure serial structure, while use the stiffness matrices for the flexure parallel structure. To achieve motion, the stiffness terms along the motion should be small, while the other stiffness terms should be large. Compliance/stiffness modeling can represent the relationship between the compliance/stiffness and geometrical parameters, and thus it can be applied to optimize the geometrical parameters. Therefore, Compliance/stiffness modeling methods are crucial to design planar flexure-based compliant mechanisms with finite deformations.

\section{Output decoupling property}

To analyze the output decoupling property, the influence of symmetrical configuration could be investigated. For a notch flexure hinge, there are strong couplings between the rotational and translational motions. Furthermore, the rotation center of a flexure hinge drifts whenever the hinge works, resulting in motion errors. To obtain decoupling properties, multiple flexure hinges can be combined to form certain structures (Qin et al., 2013). In practice, the parallelogram joints are usually used as prismatic joints. The parallelogram joints can keep the orientation of the platform invariant when the mechanism is actuated by the force $F_{x}$ along one axis. However, undesired cross-coupling error $e_{y}$ along the other axis is generated at the same time, as depicted in Fig. 2a, which makes it difficult for the kinematic analysis and control of such a mechanism. Compared with one parallelogram joint, two parallelogram joints shown in Fig. 2b can eliminate the coupling error completely, and thus the symmetric 
configuration could be regarded as two ideal prismatic joints to form certain flexure-based compliant structures to obtain output decoupling property (Yong and Lu, 2008). Obviously, the motion illustrated in Fig. 2a is a pure translation. By contrast, the motion in Fig. $2 b$ is a pure translation without undesired cross-coupling errors. Therefore, flexure-based compliant mechanisms are usually designed with symmetric configuration to obtain the output decoupling property.

\subsection{Planar flexure-based compliant mechanisms with symmetric configuration}

\subsubsection{Symmetric configuration about $x$ axis}

As shown in Fig. 3, the planar flexure-based compliant mechanism is symmetric about the $x$ axis and all ends are fixed. We can see that the mechanism is composed of one platform and four limbs, and each limb is composed of several notch flexure hinges and rigid beams. The output point is located at the center of the platform.

The mechanism can be divided into two identical parts: the upper part and the lower part. $\mathbf{J}_{F s}$ and $\mathbf{J}_{P s}$ denote the Jacobian matrices transforming forces/moments and deformations of the upper part, $\mathbf{K}_{1}$ denotes the stiffness matrix at the point $o$ of half part $\left(K_{1, m-n}\right.$ represents the stiffness in the direction of $m$ caused by the force/moment $n$ ), while $\mathbf{J}_{F x}$ and $\mathbf{J}_{P x}$ are defined as the Jacobian matrices transforming forces/moments and deformations of the lower part. Then, the stiffness matrices of the upper part and lower part can be given as

$\mathbf{K}_{t s}=\mathbf{J}_{F s} \cdot \mathbf{K}_{1} \cdot \mathbf{J}_{P s}=\mathbf{K}_{1}$,

$\mathbf{K}_{t x}=\mathbf{J}_{F x} \cdot \mathbf{K}_{1} \cdot \mathbf{J}_{P x}$,

where

$\mathbf{J}_{F s}=\mathbf{J}_{P s}^{T}=\mathbf{E}$ (identity matrix),

$\mathbf{K}_{1}=\left[\begin{array}{lll}K_{1, x-F x} & K_{1, x-F y} & K_{1, x-M z} \\ K_{1, y-F x} & K_{1, y-F y} & K_{1, y-M z} \\ K_{1, \alpha-F x} & K_{1, \alpha-F y} & K_{1, \alpha-M z}\end{array}\right]$,

and

$\mathbf{J}_{F x}=\mathbf{J}_{P x}^{T}=\left[\begin{array}{ccc}1 & 0 & 0 \\ 0 & -1 & 0 \\ 0 & 0 & -1\end{array}\right]$.

Thus, the stiffness matrix of the flexure-based compliant mechanism is given as

$\mathbf{K}=\mathbf{K}_{t s}+\mathbf{K}_{t x}=\left[\begin{array}{ccc}2 K_{1, x-F x} & 0 & 0 \\ 0 & 2 K_{1, y-F y} & 2 K_{1, y-M z} \\ 0 & 2 K_{1, \alpha-F y} & 2 K_{1, \alpha-M z}\end{array}\right]$.

We can see from Eq. (10) that the terms $K_{1, y-F x}$ and $K_{1, \alpha-F x}$ are both zero. It indicates that the flexure-based compliant mechanisms with symmetric configuration about the $x$ axis are output decoupled along the $x$ axis.
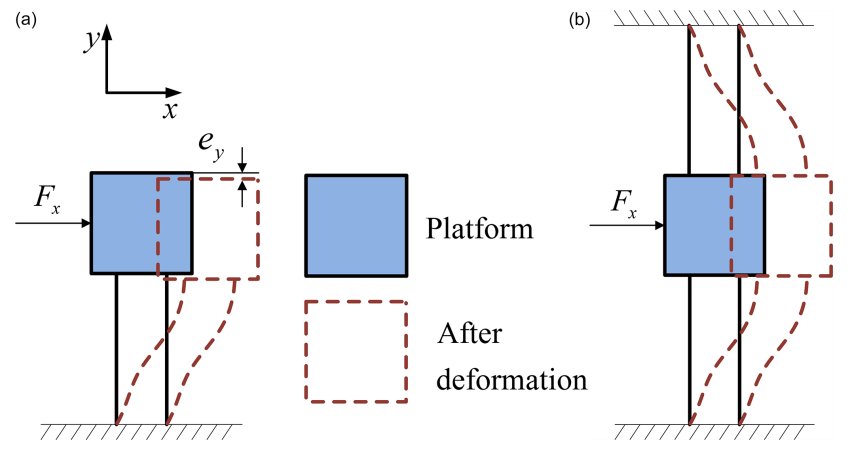

Figure 2. Parallelogram joints for planar parallel mechanisms: (a) one parallelogram joint; (b) two parallelogram joints with symmetric configuration.

\subsubsection{Symmetric configuration about $y$ axis}

Figure 4 illustrates the flexure-based compliant mechanism which is symmetric about the $y$ axis and all ends are fixed. The mechanism consists of one platform and four limbs, and the output point is located at the center of the platform.

The mechanism can be divided into two identical parts: the left part and the right part. $\mathbf{J}_{F l}$ and $\mathbf{J}_{P l}$ denote the Jacobian matrices transforming forces/moments and deformations of the left part, $\mathbf{K}_{2}$ denotes the stiffness matrix at the point $o$ of half part ( $K_{2, m-n}$ represents the stiffness in the direction of $m$ caused by the force/moment $n$ ), whereas $\mathbf{J}_{F r}$ and $\mathbf{J}_{P r}$ are defined as the Jacobian matrices transforming forces/moments and deformations of the right part. Then, the stiffness matrices of the left part and right part can be given as

$$
\begin{aligned}
& \mathbf{K}_{t l}=\mathbf{J}_{F l} \cdot \mathbf{K}_{2} \cdot \mathbf{J}_{P l}, \\
& \mathbf{K}_{t r}=\mathbf{J}_{F r} \cdot \mathbf{K}_{2} \cdot \mathbf{J}_{P r}=\mathbf{K}_{2},
\end{aligned}
$$

where

$$
\begin{aligned}
\mathbf{J}_{F l} & =\mathbf{J}_{P l}^{T}=\left[\begin{array}{ccc}
-1 & 0 & 0 \\
0 & 1 & 0 \\
0 & 0 & -1
\end{array}\right], \\
\mathbf{K}_{2} & =\left[\begin{array}{ccc}
K_{2, x-F x} & K_{2, x-F y} & K_{2, x-M z} \\
K_{2, y-F x} & K_{2, y-F y} & K_{2, y-M z} \\
K_{2, \alpha-F x} & K_{2, \alpha-F y} & K_{2, \alpha-M z}
\end{array}\right],
\end{aligned}
$$

and

$\mathbf{J}_{F r}=\mathbf{J}_{P r}^{T}=\mathbf{E}$ (identity matrix).

Thus, the stiffness matrix of the mechanism can be expressed as

$\mathbf{K}=\mathbf{K}_{t l}+\mathbf{K}_{t r}=\left[\begin{array}{ccc}2 K_{2, x-F x} & 0 & 2 K_{2, x-M z} \\ 0 & 2 K_{2, y-F y} & 0 \\ 2 K_{2, \alpha-F x} & 0 & 2 K_{2, \alpha-M z}\end{array}\right]$.

We can see from Eq. (13) that the terms $K_{2, x-F y}$ and $K_{2, \alpha-F y}$ are both zero. It indicates that the flexure-based compliant mechanisms with symmetric configuration about the $y$ axis are output decoupled along the $y$ axis. 


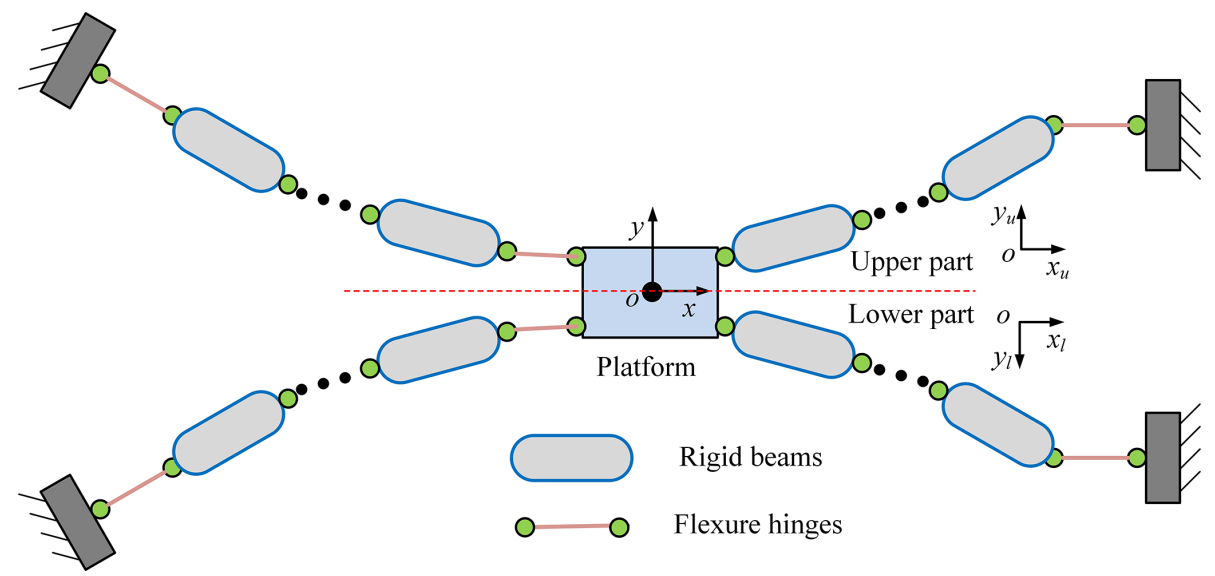

Figure 3. Mechanism with symmetric configuration about the $x$ axis.

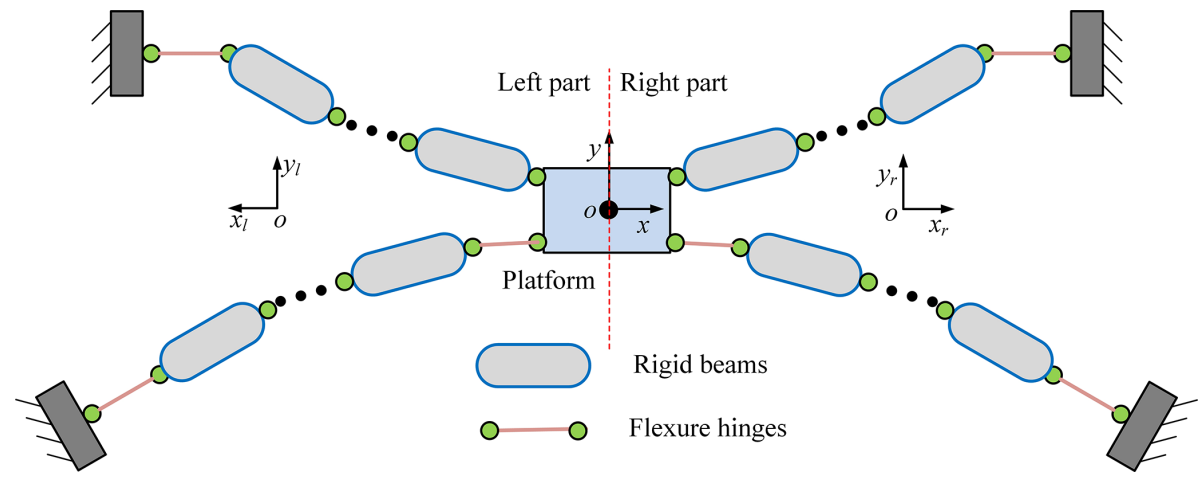

Figure 4. Mechanism with symmetric configuration about the $y$ axis.

\subsubsection{Symmetric configuration about $x$ axis and $y$ axis}

The flexure-based compliant mechanism is symmetric about two perpendicular axes (the $x$ axis and $y$ axis), and the output point is located at the center of the platform. The flexurebased compliant mechanism can be divided into two identical parts: the left part and the right part. Denote $\mathbf{K}_{2}$ as the stiffness matrix at the point $o$ of half part $\left(K_{2, m-n}\right.$ is the stiffness in the direction of $m$ caused by the force/moment $n$ ), the stiffness matrix of the flexure-based compliant mechanism can be expressed as

$$
\begin{aligned}
\mathbf{K} & =\mathbf{J}_{F l} \cdot \mathbf{K}_{2} \cdot \mathbf{J}_{P l}+\mathbf{J}_{F r} \cdot \mathbf{K}_{2} \cdot \mathbf{J}_{P r} \\
& =\left[\begin{array}{ccc}
2 K_{2, x-F x} & 0 & 0 \\
0 & 2 K_{2, y-F y} & 0 \\
0 & 0 & 2 K_{2, \alpha-M z}
\end{array}\right] .
\end{aligned}
$$

We can see from Eq. (14) that the stiffness matrix consists of only main diagonal components. Therefore, the analyses above indicate that the planar flexure-based compliant mechanisms are output decoupled when they are symmetric about two perpendicular axes (the $x$ axis and $y$ axis).

\subsection{Variations of the fundamental form}

\subsubsection{The fundamental form}

The fundamental form is composed of several flexure hinges, and it can be regarded as the limb of flexure parallel structures. The fundamental form can be extended according to its designated function, such as the parallelogram joints and prismatic joints mentioned above. In general, the mechanisms may be composed of several fundamental forms. In order to analyze the performances of flexure-based compliant mechanisms, the fundamental form, which is actually a flexure serial structure, should be first investigated. In practice, the notch flexure hinges of the flexure serial structure are all along one straight line, as shown in Fig. 5.

According to compliance/stiffness modeling methods discussed above, the compliance of the practical flexure serial structure can be obtained from Eq. (4), and $\mathbf{J}_{d i}, \mathbf{J}_{f i}$, and $\mathbf{C}_{i}^{l}$ can be given as

$\mathbf{J}_{d i}=\mathbf{J}_{f i}^{T}=\left[\begin{array}{ccc}1 & 0 & r_{y} \\ 0 & 1 & -r_{x} \\ 0 & 0 & 1\end{array}\right]\left[\begin{array}{ccc}-1 & 0 & 0 \\ 0 & 1 & 0 \\ 0 & 0 & -1\end{array}\right]$, 


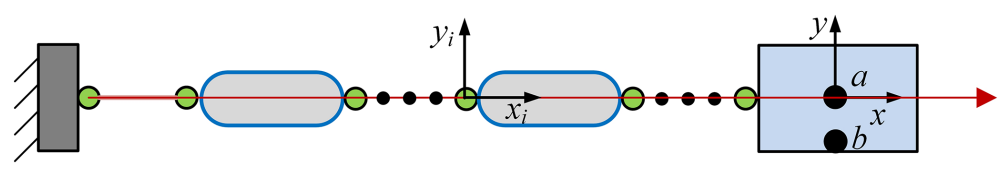

Figure 5. The practical flexure serial structure.

$\mathbf{C}_{i}^{l}=\left[\begin{array}{ccc}C_{i, x-F x} & 0 & 0 \\ 0 & C_{i, y-F y} & C_{i, y-M z} \\ 0 & C_{i, \alpha-F y} & C_{i, \alpha-M z}\end{array}\right]$,

where $r_{x}$ and $r_{y}$ are the distances from the platform at the reference point to the $i$ th flexure hinge along the $x$ axis and $y$ axis, respectively, $C_{i, m-n}$ is the compliance of $i$ th hinge in the direction of $m$ caused by the force/moment $n$.

Then, when the output point of the structure is located at the reference point $b$, the compliance of the flexure serial structure can be given as

$\mathbf{C}_{b}=\sum_{i=1}^{n} \mathbf{J}_{d i} \mathbf{C}_{i}^{l} \mathbf{J}_{f i}=\left[\begin{array}{lll}C_{b, x-F x} & C_{b, x-F y} & C_{b, x-M z} \\ C_{b, y-F x} & C_{b, y-F y} & C_{b, y-M z} \\ C_{b, \alpha-F x} & C_{b, \alpha-F y} & C_{b, \alpha-M z}\end{array}\right]$,

where

$C_{b, x-F x}=\sum_{i=1}^{n}\left(C_{i, x-F x}+r_{y}^{2} C_{i, \alpha-M z}\right)$,

$C_{b, y-F y}=\sum_{i=1}^{n}\left(C_{i, y-F y}+2 r_{x} C_{i, y-M z}+r_{x}^{2} C_{i, \alpha-M z}\right)$,

$C_{b, y-M z}=\sum_{i=1}^{n}\left(-C_{i, y-M z}+r_{x} C_{i, \alpha-M z}\right)$

and

$C_{b, \alpha-M z}=\sum_{i=1}^{n}\left(C_{i, \alpha-M z}\right)$.

When the output point of the structure is located at the reference point $a$, the distance $r_{y}$ is zero, and the compliance of the flexure serial structure can be given as

$\mathbf{C}_{a}=\sum_{i=1}^{n} \mathbf{J}_{d i} \mathbf{C}_{i}^{l} \mathbf{J}_{f i}=\left[\begin{array}{ccc}C_{a, x-F x} & 0 & 0 \\ 0 & C_{a, y-F y} & C_{a, y-M z} \\ 0 & C_{a, \alpha-F y} & C_{a, \alpha-M z}\end{array}\right]$,

where

$C_{a, x-F x}=\sum_{i=1}^{n} C_{i, x-F x}$,

$C_{a, y-F y}=C_{b, y-F y}$,

$C_{a, y-M z}=C_{b, y-M z}$

and

$C_{a, \alpha-M z}=C_{b, \alpha-M z}$.

Therefore, the analyses show that the compliance/stiffness matrix can be expressed as Eq. (18) when the flexure hinges and output reference point are all along one straight line, and it can be expressed as Eq. (17) when the flexure hinges and output reference point are not along one straight line.

\subsubsection{Variations}

Besides the symmetric configuration about $x$ axis and $y$ axis, without loss of generality, the flexure-based compliant mechanisms may be composed of several identical fundamental forms which are distributed evenly around the center of the platform, as shown in Fig. 6 (Yong and Lu, 2008). When the output point is located at the center of the platform and the number of the fundamental forms is $2 n$ or 3 , the output decoupling of them could be discussed as follows.

As the analyses mentioned above, the mechanism can be divided into $2 n(n>1)$ identical fundamental forms. Denote $\mathbf{K}_{m}$ as the stiffness matrix at the point $o$ of one fundamental form $\left(K_{m, w-u}\right.$ represents the stiffness in the direction of $w$ caused by the force/moment $u$ ). When the flexure hinges and output reference point of one fundamental form are along one straight line, the stiffness matrix of the $i$ th fundamental form can be given as

$\mathbf{K}_{i}=\mathbf{J}_{F i} \cdot \mathbf{K}_{m} \cdot \mathbf{J}_{P i}$,

where

$\begin{aligned} \mathbf{K}_{m} & =\left[\begin{array}{ccc}K_{m, x-F x} & 0 & 0 \\ 0 & K_{m, y-F y} & K_{m, y-M z} \\ 0 & K_{m, \alpha-F y} & K_{m, \alpha-M z}\end{array}\right], \\ \mathbf{J}_{F i} & =\left[\begin{array}{ccc}\cos [(i-1) \pi / n] & \sin [(i-1) \pi / n] & 0 \\ -\sin [(i-1) \pi / n] & \cos [(i-1) \pi / n] & 0 \\ 0 & 0 & 1\end{array}\right],\end{aligned}$

and

$\mathbf{J}_{P i}=\left[\begin{array}{ccc}\cos [(i-1) \pi / n] & -\sin [(i-1) \pi / n] & 0 \\ \sin [(i-1) \pi / n] & \cos [(i-1) \pi / n] & 0 \\ 0 & 0 & 1\end{array}\right]$.

Hence, the stiffness matrix of the mechanism at center of the platform is given as

$\mathbf{K}_{2 n}=\sum_{1}^{2 n} \mathbf{K}_{i}$

$=\left[\begin{array}{ccc}n K_{m, x-F x}+n K_{m, y-F y} & 0 & 0 \\ 0 & n K_{m, y-F y}+n K_{m, x-F x} & 0 \\ 0 & 0 & 2 n K_{m, \alpha-M z}\end{array}\right]$.

We can see from Eq. (20) that the stiffness matrix is a diagonal matrix. It indicates that the flexure-based compliant mechanisms composed of $2 n(n>1)$ fundamental forms are output decoupled. The stiffness matrix of the mechanism can be obtained according to Eq. (20) when the number of the 
(a)

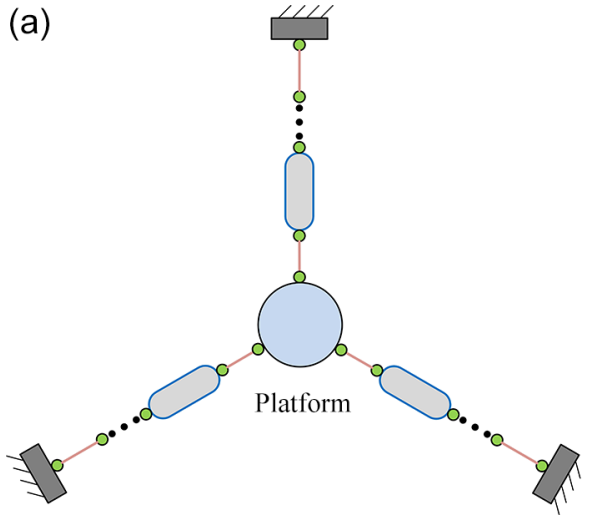

(b)

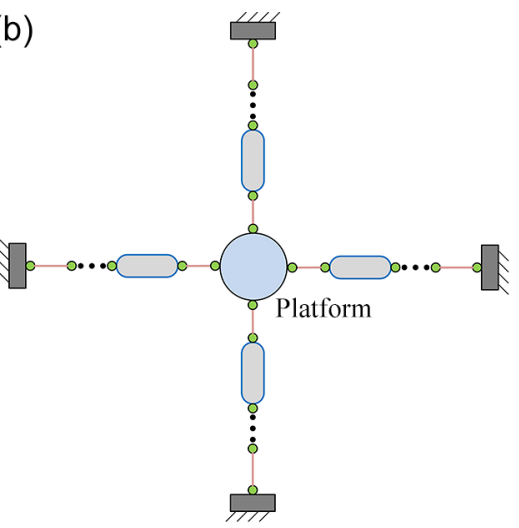

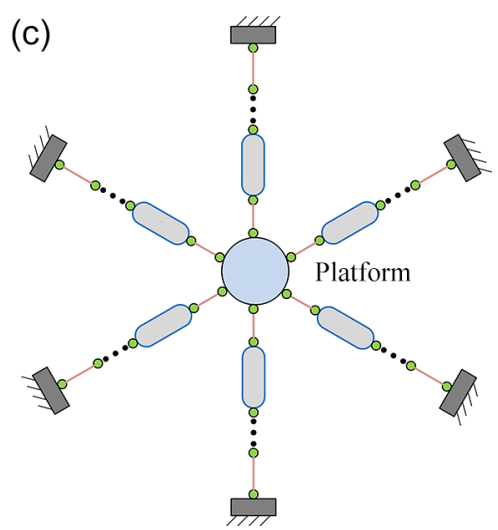

Figure 6. Fundamental forms distributed evenly around the center of the platform: (a) $n=3$; (b) $n=4$; (c) $n=6$.

fundamental forms is 4 and 6, as shown in Fig. 7b and c. In addition, there are some special conditions should be taken into account. When the number of the fundamental forms is 3, as shown in Fig. 7a, the stiffness matrix of the mechanism can be obtained from Eq. (19), and it can be expressed as

$$
\begin{aligned}
& \mathbf{K}_{3}=\sum_{1}^{3} \mathbf{K}_{i} \\
& =\left[\begin{array}{ccc}
\frac{3}{2} K_{m, x-F x}+\frac{3}{2} K_{m, y-F y} & 0 & 0 \\
0 & \frac{3}{2} K_{m, y-F y}+\frac{3}{2} K_{m, x-F x} & 0 \\
0 & 0 & 3 K_{m, \alpha-M z}
\end{array}\right] .
\end{aligned}
$$

Similarly, we can see from Eq. (21) that the stiffness matrix is a diagonal matrix. It indicates that the flexure-based compliant mechanisms composed of 3 fundamental forms are output decoupled. In addition, when the mechanism is symmetric about $y$ axis and the number of the fundamental forms is 2 , the stiffness matrix of the mechanism can be obtained from Eq. (14), and it can be expressed as

$\mathbf{K}_{2}=\sum_{1}^{2} \mathbf{K}_{i}=\left[\begin{array}{ccc}2 K_{m, x-F x} & 0 & 0 \\ 0 & 2 K_{m, y-F y} & 0 \\ 0 & 0 & 2 K_{m, \alpha-M z}\end{array}\right]$.

Therefore, the flexure-based compliant mechanisms composed of $2 n$ or 3 identical fundamental forms which are distributed evenly around the center of the platform are output decoupled. In addition, the stiffness of mechanisms composed of $2 n(n>1)$ or 3 fundamental forms can be calculated from Eqs. (20) and (21), while the stiffness of mechanisms composed of 2 fundamental forms which are symmetric about $y$ axis can be calculated from Eq. (22).

\section{Application}

To validate the analytical model derived above, simple closed-form equations are derived to determine the output compliances of the RRR and 4-RRR stages (Yong and Lu, 2008). The RRR flexure-based compliant structure is composed of three constant rectangular cross-section flexure

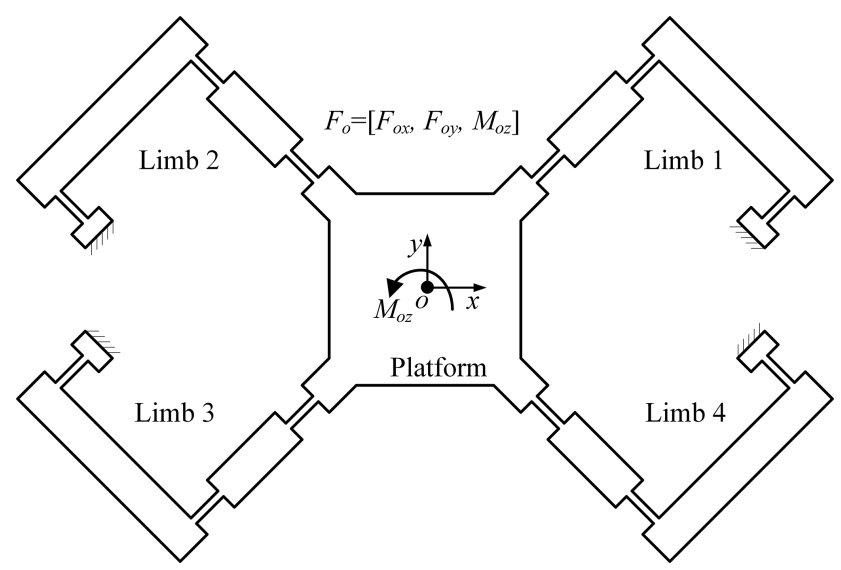

Figure 7. 4-RRR flexure-based compliant micro-motion stage.

hinges, and the 4-RRR structure is composed of four RRR limbs connected together in parallel, as shown in Fig. 7. The parallel configuration of the 4-RRR structure is advantageous over a serial configuration structure.

\subsection{Output compliance of the RRR micro-motion stage}

Notch flexure hinges are produced by drilling or milling two closely spaced holes, forming a circular or constant rectangular cross-section cutout (Tseytlin, 2002). The geometrical parameters of the constant rectangular cross-section flexure hinge are illustrated in Fig. 8. The geometrical dimensions include the hinge thickness $t$, the hinge length $L$, the side height $h$, the rigid beam width $W$, the total length $L_{a}$, the total height $H$, and the depth $D$.

The in-plane compliance matrix of the constant rectangular cross-section flexure hinge is shown in Eq. (16). The exponential relationship between geometrical parameters and deformations can be derived through FEA, and the proposed compliance matrix can be obtained based on the exponential model. The compliance terms can be expressed as follows. 


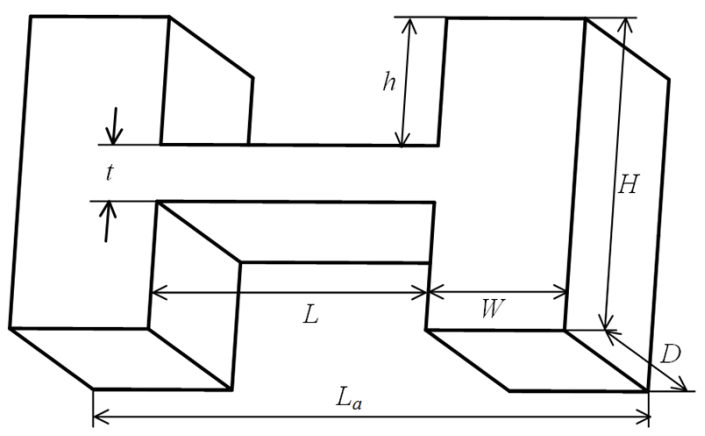

Figure 8. Geometrical dimensions of the constant rectangular cross-section flexure hinge.

$C_{i, x-F x}=\frac{4}{E D H \cdot t^{0.82}}\left[h^{1.07} \cdot L^{0.75}+L_{a}\right]$

$C_{i, \alpha-M z}=\frac{16.5 \cdot L^{0.88}}{E D \cdot t^{2.88}}$

$C_{i, y-M z}=C_{i, \alpha-F y}=\frac{8.3 \cdot L^{1.88}}{E D \cdot t^{2.88}}$

$C_{i, y-F y}=\frac{5.3 \cdot h^{0.01} \cdot L^{2.9}}{E D \cdot t^{2.91}}+\frac{\alpha \cdot E \cdot C_{i, x-F x}}{G}$

where $E$ is the Yong's modulus and $\alpha$ is the shear coefficient.

Note that all flexure hinges are modeled to have 3-DOF (i.e. bending compliance about the $z$ axis, and axial and shear compliances along the $x$ and $y$ axes, respectively). Flexure hinges are modeled as having 3-DOF rather than 6-DOF because both the RRR and 4-RRR compliant stages considered here are planar. The RRR flexure-based compliant stage is shown in Fig. 9, together with its dimensions, displacements, local coordinates of flexure hinges, and the applied forces/moments.

The compliances at point $o_{1}$ (Fig. 9a) contributed by each flexure hinge in the structure are firstly calculated. The overall compliances of the RRR compliant stage at point $o$ (Fig. 9b) are then obtained by summing all the contributions to the compliances in the corresponding directions of each individual flexure hinge. The compliance matrix of each flexure hinge is shown in Eq. (16), and the geometrical parameters are illustrated in Fig. 8. Considering that $b_{o}$ and $h_{o}$ are the key distances of the RRR stage, the output compliance matrix at $o_{1}$ can be expressed as

$\mathbf{C}_{O 1}=\sum_{i=1}^{3} \mathbf{J}_{d i} \mathbf{C}_{i}^{l} \mathbf{J}_{f i}$

When the output forces are applied to point $o$ rather than $o_{1}$, and the displacements at this point are desired, the transformation matrix $\mathbf{J}_{f o}$ can be used to transfer the output forces/moments from $o_{1}$ to $o$, while $\mathbf{J}_{d o}$ can be used to transfer the displacements from $o_{1}$ to $o$, where $b_{1}$ and $h_{1}$ are the components of the vector $r$ pointing from $o_{1}$ to $o$ along
Table 1. Geometrical parameters and material properties of the compliant micro-motion stage.

\begin{tabular}{ll}
\hline $\begin{array}{l}\text { Parameters/material } \\
\text { properties }\end{array}$ \\
\hline Young's modulus, $E(\mathrm{GPa})$ & 210 \\
Poisson's ratio, $v$ & 0.30 \\
$h_{o}(\mathrm{~mm})$ & 15 \\
$b_{o}(\mathrm{~mm})$ & 50 \\
$h_{1}(\mathrm{~mm})$ & 25 \\
$b_{1}(\mathrm{~mm})$ & 25 \\
$L(\mathrm{~mm})$ & 10 \\
$t(\mathrm{~mm})$ & 2 \\
$h(\mathrm{~mm})$ & 4 \\
$W(\mathrm{~mm})$ & 5 \\
$D(\mathrm{~mm})$ & 10 \\
\hline
\end{tabular}

$x$ axis and $y$ axis, respectively. Thus, the output compliance at point $o$ can be expressed as

$\mathbf{C}_{\mathrm{RRR}, 1 O}=\mathbf{J}_{d o} \mathbf{C}_{O 1} \mathbf{J}_{f o}$,

where

$\mathbf{J}_{d o}=\mathbf{J}_{f o}^{T}=\left[\begin{array}{ccc}1 & 0 & -h_{1} \\ 0 & 1 & b_{1} \\ 0 & 0 & 1\end{array}\right]$.

\subsection{Output compliance of the 4-RRR micro-motion stage}

The 4-RRR compliant micro-motion stage is generated by arranging the four RRR compliant stages $90^{\circ}$ apart, and it is symmetric about $x$ axis and $y$ axis respectively, as shown in Fig. 7. According to the symmetric configuration, the output compliance matrix at point $o$ of limbs 2, 3 and 4, respectively, are

$\mathbf{C}_{\mathrm{RRR}, 2 O}=\mathbf{J}_{2} \mathbf{C}_{\mathrm{RRR}, 1 O} \mathbf{J}_{2}^{T}$,

$\mathbf{C}_{\mathrm{RRR}, 3 O}=\mathbf{J}_{\pi} \mathbf{C}_{\mathrm{RRR}, 1 O} \mathbf{J}_{\pi}^{T}$,

$\mathbf{C}_{\mathrm{RRR}, 4 O}=\mathbf{J}_{\pi} \mathbf{C}_{\mathrm{RRR}, 2 O} \mathbf{J}_{\pi}^{T}$,

where

$\mathbf{J}_{2}=\left[\begin{array}{ccc}-1 & 0 & 0 \\ 0 & 1 & 0 \\ 0 & 0 & -1\end{array}\right]$

and

$\mathbf{J}_{\pi}=\left[\begin{array}{ccc}\cos (\pi) & -\sin (\pi) & 0 \\ \sin (\pi) & \cos (\pi) & 0 \\ 0 & 0 & 1\end{array}\right]$.

Then, the compliance matrix of the 4-RRR flexure-based compliant micro-motion stage can be given as

$\mathbf{C}_{4 \mathrm{RRR}}=\left(\mathbf{C}_{\mathrm{RRR}, 1 O}^{-1}+\mathbf{C}_{\mathrm{RRR}, 2 O}^{-1}+\mathbf{C}_{\mathrm{RRR}, 3 O}^{-1}+\mathbf{C}_{\mathrm{RRR}, 4 O}^{-1}\right)^{-1}$. 


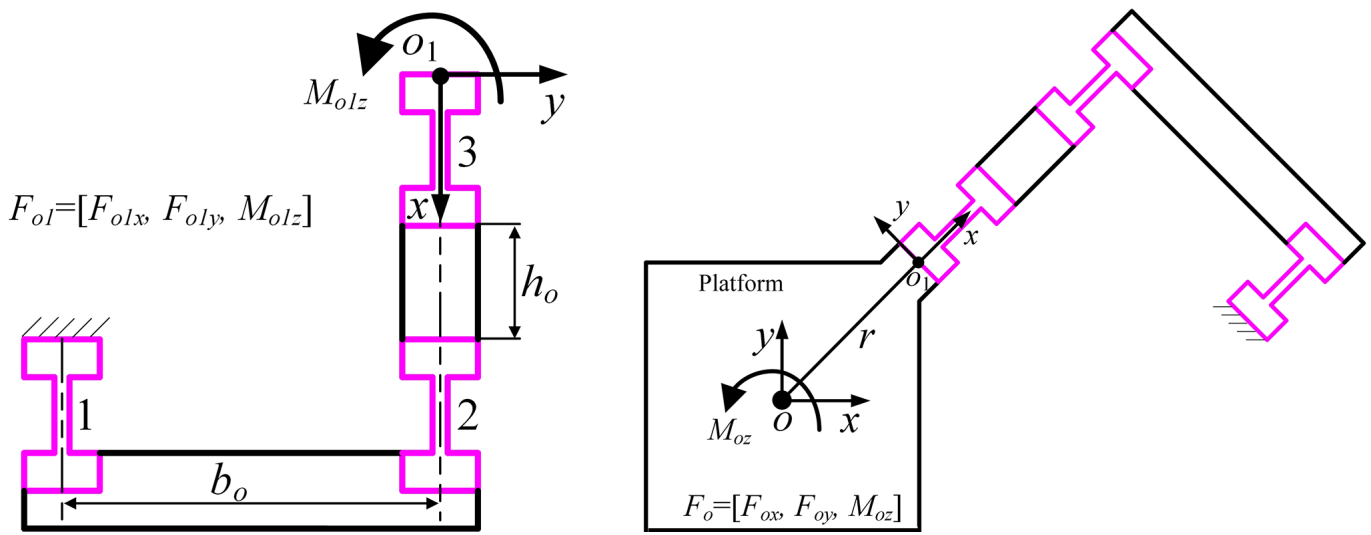

Figure 9. RRR flexure-based compliant mechanism: (a) applied forces/moments at output point $o_{1}$; (b) applied forces/moments at output point $o$.

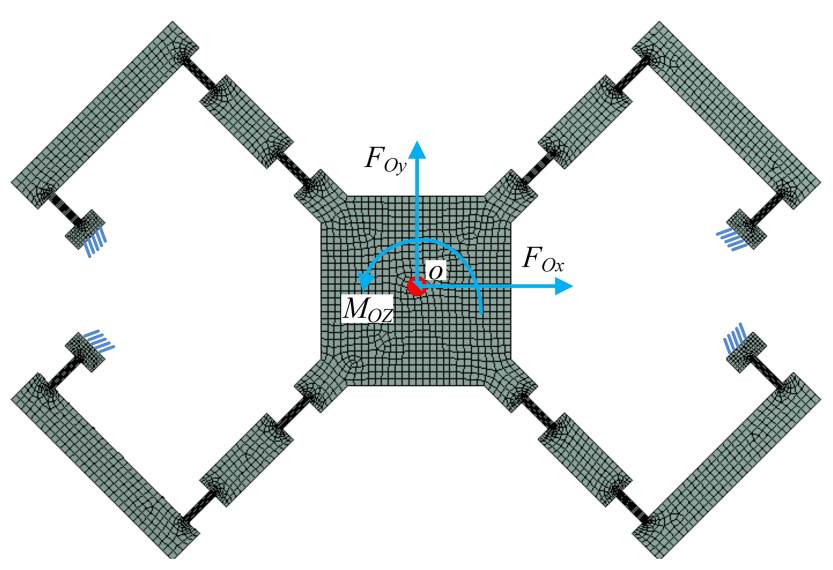

Figure 10. FEA model of a 4-RRR flexure-based compliant micromotion stage.

\subsection{Validation}

Considering the accuracy of FEA, it is used as a benchmark. The output compliances of the RRR and 4-RRR micromotion stages are calculated to study the output decoupling property. The results calculated by the analytical model are compared with those derived from FEA. Table 1 lists the geometrical parameters and material properties of the RRR and 4-RRR compliant micro-motion stages employed, where all flexure hinges have equivalent geometrical parameters and material properties. A FEA model was generated using ANSYS. Meshes, constraints, and the applied forces/moments of the FEA model are shown in Fig. 10.

The output compliance of the RRR and 4-RRR micromotion stages determined using FEA can be expressed as, respectively

$$
\mathbf{C}_{\text {RRR,FEA }}=\left[\begin{array}{ccc}
3.820 \times 10^{-5} & -5.057 \times 10^{-5} & 8.889 \times 10^{-4} \\
-5.057 \times 10^{-5} & 1.029 \times 10^{-4} & -1.457 \times 10^{-3} \\
8.889 \times 10^{-4} & -1.457 \times 10^{-3} & 2.410 \times 10^{-2}
\end{array}\right],
$$

$$
\mathbf{C}_{4 R R R, F E A}=\left[\begin{array}{ccc}
1.184 \times 10^{-6} & 0 & 0 \\
0 & 3.239 \times 10^{-6} & 0 \\
0 & 0 & 3.076 \times 10^{-4}
\end{array}\right] .
$$

Since the off-diagonal compliances terms in Eq. (34) are very small and insignificant compared to the diagonal terms, they are assumed to be zero. It indicates that deformations occur only along the direction of the applied force/moments.

Figure 11 shows the FEA results of the force-displacement relationship of the 4RRR micro-motion stage. It can be seen that the displacement of the platform along $x$ axis increases linearly with the increase of the $x$ axis actuating force. Furthermore, the displacement of $x$ axis is constant (approaching zero) when the actuation force of $y$ axis increases, while the displacement of $y$ axis is constant (approaching zero) when the actuation force of $x$ axis increases. It indicates that the 4-RRR micro-motion stage has output decoupling property, which agrees with the analyses above.

Compared with Eqs. (33) and (34), the output compliance of the RRR and 4-RRR micro-motion stages determined using analytical model can be expressed as, respectively

$$
\begin{gathered}
\mathbf{C}_{\mathrm{RRR}}=\left[\begin{array}{ccc}
3.938 \times 10^{-5} & -5.200 \times 10^{-5} & 9.161 \times 10^{-4} \\
-5.200 \times 10^{-5} & 1.058 \times 10^{-4} & -1.499 \times 10^{-3} \\
9.161 \times 10^{-4} & -1.499 \times 10^{-3} & 2.482 \times 10^{-2}
\end{array}\right], \\
\mathbf{C}_{4 \mathrm{RRR}}=\left[\begin{array}{ccc}
1.205 \times 10^{-6} & 0 & 0 \\
0 & 3.273 \times 10^{-6} & 0 \\
0 & 0 & 3.096 \times 10^{-4}
\end{array}\right] .
\end{gathered}
$$

We can see from Eq. (36) that the compliance matrix is a diagonal matrix, and it indicates that the 4-RRR compliant micro-motion stage is output decoupled. Analytical output compliances were compared with those derived from FEA. The differences between the analytical and FEA results are shown in Table 2.

It is found that values obtained from the proposed analytical model are almost similar to the FEA values of the RRR and 4-RRR compliant micro-motion stages, which validates the proposed analytical model. 
Table 2. The differences between the analytical and FEA results.

\begin{tabular}{|c|c|c|c|c|c|c|c|c|}
\hline \multicolumn{3}{|c|}{ Output compliance matrix } & \multicolumn{3}{|c|}{$\%$ difference of RRR } & \multicolumn{3}{|c|}{$\%$ difference of 4-RRR } \\
\hline$C_{x-F x}$ & $C_{y-F x}$ & $C_{\alpha-F x}$ & 3.08 & 2.83 & 3.05 & 1.77 & - & - \\
\hline$C_{x-F y}$ & $C_{y-F y}$ & $C_{\alpha-F y}$ & 2.83 & 2.83 & 2.88 & - & 1.05 & - \\
\hline$C_{X-M z}$ & $C_{y-M z}$ & $C_{\alpha-M z}$ & 3.05 & 2.88 & 2.98 & - & - & 0.65 \\
\hline
\end{tabular}
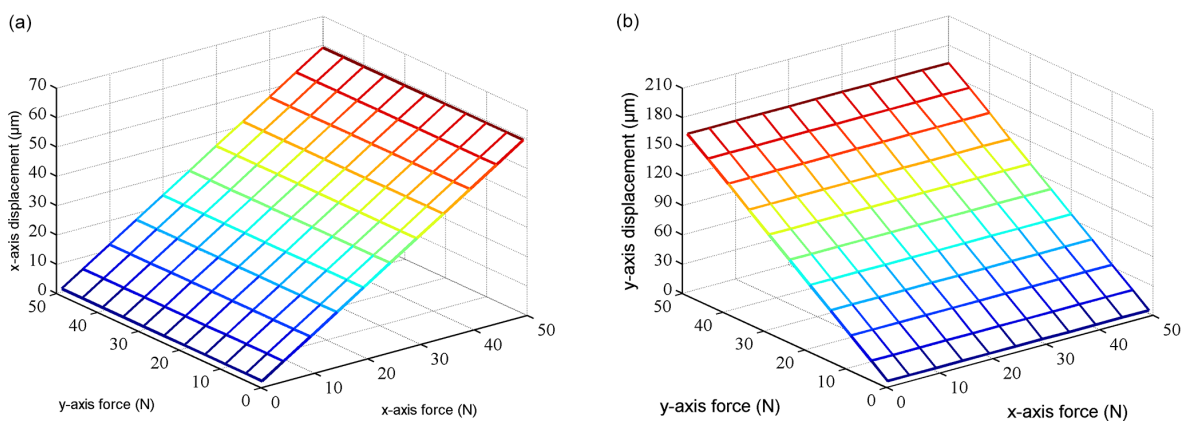

Figure 11. FEA results of force-displacement relationship: (a) displacement of $x$ axis; (b) displacement of $y$ axis.

\section{Conclusions}

This paper presented the output decoupling property of planar flexure-based compliant mechanisms. The compliance/stiffness modeling methods were first derived. Then, analytical model utilized for analyzing the output decoupling property of flexure-based compliant mechanisms was obtained in terms of the influences of symmetric configuration. It involved the mechanisms which were symmetric about two perpendicular axes or composed of several fundamental forms. To validate the output decoupling property, the output compliances of the RRR and 4-RRR compliant micro-motion stages were analyzed with the proposed analytical model and FEA. Based upon the results obtained, we can draw the following conclusions:

1. flexure-based compliant mechanisms are output decoupled when they are symmetric about two perpendicular axes;

2. flexure-based compliant mechanisms composed of either three or an even number of identical fundamental forms distributed evenly around the center are output decoupled;

3. comparisons with the results obtained by FEA demonstrate that the proposed analytical model of the 4-RRR micro-motion stage with symmetric configuration is valid.
Acknowledgements. This work was supported by the National Basic Research Program of China (Grant No. 2011CB302400), the National Science Foundation of China (Grant No. 51275260), and the National Science and Technology Major Project of China (Grant No. 2015ZX04001002).

Edited by: K. Mianowski

Reviewed by: two anonymous referees

\section{References}

Acer, M. and Sabanovic, A.: Comparison of circular flexure hinge compliance modeling methods, in: Proceedings of the 2011 IEEE International Conference on Mechatronics, 13-15 April 2011, Istanbul, Turkey, 271-276, 2011.

Ando, T., Kodera, N., Maruyama, D., Takai, E., Saito, K., and Toda, A.: A high-speed atomic force microscope for studying biological macromolecules in action, Jpn. J. Appl. Phys/, 41, 48514856, doi:10.1143/JJAP.41.4851, 2002.

Awtar, S. and Slocum, A. H.: Constraint-based design of parallel kinematic $X Y$ flexure mechanisms, J. Mech. Des., 129, 816-830, doi:10.1115/1.2735342, 2007.

Choi, K.-B. and Lee, J. J.: Passive compliant wafer stage for singlestep nano-imprint lithography, Rev. Sci. Instrum., 76, 075106, doi:10.1063/1.1948401, 2005.

Handley, D. C., Lu, T.-F., Yong, Y. K., and Hales, C.: Workspace investigation of a 3 DOF compliant micro-motion stage, in: Proceedings of the International Conference on Control, Automation, Robotics and Vision Conference, 6-9 December 2004, Kunming, China, 1279-1284, 2004.

Kenton, B. J. and Leang, K. K.: Design and control of a three-axis serial-kinematic high-bandwidth nanopositioner, IEEE/ASME T. Mechatron., 17, 356-369, doi:10.1109/TMECH.2011.2105499, 2012 . 
Kim, H.-Y., Ahn, D.-H., and Gweon, D.-G.: Development of a novel 3 degrees of freedom flexure based positioning system, Rev. Sci. Instrum, 83, 055114, doi:10.1063/1.4720410, 2012.

Lai, L.-J., Gu, G.-Y., and Zhu, L.-M.: Design and control of a decoupled two degree of freedom translational parallel micro-positioning stage, Rev. Sci. Instrum., 83, 045105, doi:10.1063/1.3700182, 2012.

Leang, K. K. and Fleming, A. J.: High-speed serial-kinematic SPM scanner: design and drive considerations, Asian. J. Control, 11, 144-153, doi:10.1002/asjc.090, 2009.

Li, C.-X., Gu, G.-Y., Yang, M.-J., and Zhu, L.-M.: Design, analysis and testing of a parallel-kinematic highbandwidth $X Y$ nanopositioning stage, Rev. Sci. Instrum., 84, 125111, doi:10.1063/1.4848876, 2013.

Li, Y. and Xu, Q.: Design of a new decoupled $X Y$ flexure parallel kinematic manipulator with actuator isolation, in: Proceedings of the International Conference on Intelligent Robots and Systems, 22-26 September 2008, Nice, France, 470-475, 2008.

$\mathrm{Li}, \mathrm{Y}$. and $\mathrm{Xu}, \mathrm{Q}$.: Development and assessment of a novel decoupled $X Y$ parallel micropositioning platform, IEEE/ASME T. Mechatron., 15, 125-135, doi:10.1109/TMECH.2009.2019956, 2010.

Li, Y., Huang, J., and Tang, H.: A compliant parallel $X Y$ micromotion stage with complete kinematic decoupling, IEEE T. Automat. Sci. Eng., 9, 538-553, doi:10.1109/TASE.2012.2198466, 2012.

Pham, H.-H. and Chen, I.-M.: Stiffness modeling of flexure parallel mechanism, Precis. Eng., 29, 467-478, doi:10.1016/j.precisioneng.2004.12.006, 2005.

Polit, S. and Dong, J.: Development of a high-bandwidth $\mathrm{XY}$ nanopositioning stage for high-rate micro/nanomanufacturing, IEEE/ASME T. Mechatron., 16, 724-733, doi:10.1109/TMECH.2010.2052107, 2011.

Qin, Y., Shirinzadeh, B., Zhang, D., and Tian, Y.: Compliance modeling and analysis of statically indeterminate symmetric flexure structures, Precis. Eng., 37, 415-424, doi:10.1016/j.precisioneng.2012.11.004, 2013.

Qin, Y., Shirinzadeh, B., Tian, Y., Zhang, D., and Bhagat, U.: Design and computational optimization of a decoupled 2-DOF monolithic mechanism, IEEE/ASME T. Mechatron., 19, 872881, doi:10.1109/TMECH.2013.2262801, 2014.
Schitter, G., Åström, K. J., DeMartini, B. E., Thurner, P. J., Turner, K. L., and Hansma, P. K.: Design and modeling of a high-speed AFM-scanner, IEEE T. Control Syst. Technol., 15, 906-915, doi:10.1109/TCST.2007.902953, 2007.

Tseytlin, Y. M.: Notch flexure hinges: an effective theory, Rev. Sci. Instrum., 73, 3363-3368, doi:10.1063/1.1499761, 2002.

Verma, S., Kim, W.-J., and Shakir, H.: Multi-axis maglev nanopositioner for precision manufacturing and manipulation applications, IEEE T. Indust. Appl., 41, 1159-1167, doi:10.1109/TIA.2005.853374, 2005.

Wang, H. and Zhang, X.: Input coupling analysis and optimal design of a 3-DOF compliant micropositioning stage, Mech. Mach. Theory, 43, 400-410, doi:10.1016/j.mechmachtheory.2007.04.009, 2008.

Yong, Y. K. and Lu, T.-F.: The effect of the accuracies of flexure hinge equations on the output compliances of planar micro-motion stages, Mech. Mach. Theory, 43, 347-363, doi:10.1016/j.mechmachtheory.2007.03.007, 2008.

Yong, Y. K., and Lu, T.-F.: Comparison of circular flexure hinge design equations and the derivation of empirical stiffness formulations, in: Proceedings of the 2009 IEEE International Conference on Advanced Intelligent Mechatronics, 14-17 July 2009, Singapore, 510-515, 2009.

Yong, Y. K., Lu, T.-F., and Handley, D. C.: Review of circular flexure hinge design equations and derivation of empirical formulations, Precis. Eng., 32, 63-70, doi:10.1016/j.precisioneng.2007.05.002, 2008.

Yu, J., Li, S., Su, H., and Culpepper, M. L.: Screw theory based methodology for the deterministic type synthesis of flexure mechanisms, J. Mech. Robot., 3, 031008, doi:10.1115/1.4004123, 2011.

Zubir, M. N. M., Shirinzadeh, B., and Tian, Y.: Development of a novel flexure-based compliant microgripper for high precision micro-object manipulation, Sens. Actuators A, 150, 257-266, doi:10.1016/j.sna.2009.01.016, 2009. 